\title{
Propuestas de incorporación de contenidos matemáticos de nivel superior en la educación básica: un estudio de los trabajos finales de curso del Máster Profesional en Matemáticas en la Red Nacional ${ }^{1}$
}

\begin{abstract}
Adriana Breda
Vicenç Font

Valderez Marina do Rosário Lima

Resumen

Este trabajo tiene como objetivo comprender qué papel tiene, en las propuestas de innovación para la enseñanza básica - presentadas en los trabajos de conclusión del curso (TCC) realizados en Máster Profesional en Matemáticas en la Red Nacional, Brasil -, la incorporación de contenidos matemáticos de nivel superior. Para ello, se realizó un estudio cualitativo de veintinueve trabajos, publicados durante el primer semestre de 2013 y segundo semestre de 2014 . El análisis realizado permite concluir que casi la mitad de los TCC basan su propuesta innovadora en la incorporación de contenidos de matemáticas discretas y introducción al cálculo. Las justificaciones que se dan, para este tipo de innovación en la Educación Básica, es que la incorporación de contenidos de nivel superior permite: 1) nuevas maneras de relacionar y abordar contenidos matemáticos, 2) la realización de procesos relevantes, 3 ) clases más atractivas que despiertan el interés de los alumnos.
\end{abstract} Contenidos.

Palabras clave: Máster Profesional en Matemáticas, TCC, Nuevos

\section{Abstract}

Incorporation of higher education content on basic education proposals: a study of the final course assignments of National Network Professional Master Programme in Mathematics, Brazil

The objective of this work is to understand the role of incorporation of higher educational content on innovation proposals for basic education, reported on Final Course Assignments within Brazilian Professional Master Programme in Mathematics. A qualitative study was carried out addressing a total of twenty nine works, published from the first half of 2013 to second half of 2014. It was possible to conclude that almost half of the works have their innovation proposals based on discrete mathematical content and introduction to calculation. The justification given for this kind of innovation is that the incorporation of higher educational

1 Trabajo realizado con el apoyo financiero del programa CAPES/PDSE, proceso no: 99999.004658/2014-00

136 DOI: Em andamento.

R. Bras. de Ensino de C\&T 
content allows: 1) new ways of relating and addressing mathematical content, 2) conduction of relevant mathematical processes and, 3) more attractive classes that stimulates the interest of the students.

Keywords: Professional Master in Mathematics; Final Course Assignment; New mathematical content.

\section{Introducción}

Las políticas de formación continuada realizadas por la administración educativa, de manera general, tienen por objetivo que los profesores realicen una práctica que sea cada vez mejor, de más calidad. Si bien hay diferentes políticas de formación continuada, hay dos modelos organizativos claramente diferenciados. En el primer caso, se realizan asesoramientos en el propio centro educativo, para conseguir una reflexión crítica sobre la propia práctica de la cual se puedan derivar cambios. En el segundo caso, con el objetivo de conseguir el desarrollo profesional de los docentes, se ofrecen cursos de formación permanente a los que el profesor se inscribe a título personal. Se supone que el desarrollo conseguido producirá un cambio en las prácticas del profesor asistente, que a su vez se pueden extender a sus compañeros de su centro escolar.

En relación al segundo modelo, Moreira (2004) plantea el argumento de que los másters académicos actuales no satisfacen las necesidades emergentes de la práctica docente $y$, en consecuencia, defiende la idea de la creación de másteres profesionales en la enseñanza de disciplinas específicas (Matemáticas, Física, Ciencias, etc.), ya que este tipo de máster se dirige a la práctica de los profesores y un plan de estudios que aborda la didáctica de su área del conocimiento.

En un intento de formar a los profesores de matemáticas en ejercicio, siguiendo las sugerencias del párrafo anterior y responder al objetivo dieciséis de la ley 13.005 / 2014 del Plan Nacional de Educación (PNE) - conseguir que en el año de 2020 cincuenta por ciento de los maestros de educación básica logren una formación en postgrado (BRASIL, 2014) - se inició en 2010, el Máster Profesional en Matemáticas en la Red Nacional (PROFMAT), a través de la recomendación del Consejo Técnico-Científico de Educación Superior de la Capes.

Este máster está constituido como un curso de postgrado strictu sensu, semipresencial, se ofrece en todo el territorio nacional de Brasil, está coordinado por la Sociedad Brasileña de las Matemáticas (SBM), y tiene como objetivo principal atender a los maestros de matemáticas que trabajan en la enseñanza básica, especialmente en las escuelas públicas. Su objetivo es la mejora de su formación profesional, con énfasis en el dominio profundizado del contenido matemático relevante para su desempeño docente, de acuerdo con la misión estatutaria de la SBM "estimular la mejora de la enseñanza de las matemáticas en todos los niveles." En este sentido, tiene como objetivos principales, (BRASIL, 2013a; 2013b).

1. Estimular la mejora de la enseñanza de las matemáticas en todos los niveles;

R. B. E. C. T., vol 8, núm. 3, mai-ago.2015 ISSN - 1982-873X

DOI: Em andamento. 
2. Calificar a los profesores de matemáticas que trabajan en la enseñanza básica em nivel de posgrado stricto sensu, con énfasis en el dominio profundizado de los contenidos matemáticos, ofreciendo un curso de formación profesional que se ocupa de las necesidades derivadas del trabajo diario en el cotidiano de la escuela;

3. Fomentar una actitud crítica sobre las clases de matemáticas en los niveles de educación primaria y secundaria, subrayando el papel central de los conocimientos de las matemáticas para afrontar a las demandas de la sociedad moderna;

4. Buscar el desarrollo profesional de los docentes mediante la mejora de su formación.

Con el fin de contribuir a la mejora de la enseñanza de las matemáticas, los profesores que realizan este máster deben materializar sus conocimientos en un proyecto de fin de carrera (TCC) que busque la interrelación entre el conocimiento teórico y práctico. Por esta razón, las orientaciones del PROFMAT dicen que este TCC debe tener un tratamiento inovador en la enseñanza de los temas del currículo de matemáticas de la educación básica y que, de preferencia, tenga aplicación directa de en el aula, lo que contribuye al enriquecimiento de la enseñanza de la disciplina. (BRASIL, 2013a).

En este sentido, el trabajo que aquí se presenta pretende investigar qué papel tiene, en las propuestas de innovación para la enseñanza básica - realizadas en los trabajos de conclusión del curso (TCC) realizados en Máster Profesional en Matemáticas en la Red Nacional, Brasil - la incorporación de contenidos matemáticos de nivel superior. Dado que la incorporación de nuevos contenidos es un aspecto que los profesores utilizan, muchas veces, para justificar que su propuesta es innovadora. Para ello creemos que es necesario buscar información sobre las siguientes preguntas: ¿Cuántos trabajos de conclusión de curso incorporan la introducción de contenidos matemáticos de la enseñanza superior en la educación básica? ¿Cuáles son los contenidos incluidos en estas propuestas? ¿Qué argumentos utilizan, los autores, para justificar que dicha incorporación es una mejora de la enseñanza de las matemáticas?

Aunque el Máster Profesional en Matemáticas en la Red Nacional se ofrezca en todo Brasil, el estudio realizado en este trabajo se ha restringido al análisis de los TCC en el estado de Rio Gran del Sur.

\section{Aspectos teóricos y metodológicos}

La investigación sobre las características que deben tener los programas de formación y desarrollo profesional de los profesores de matemáticas para que sean eficaces está relacionada con la idea de la mejora de la enseñanza, dado que el objetivo final de estos programas es conseguir un impacto en la mejora de la enseñanza, de las matemáticas en nuestro caso. Aparece pues el problema de lo que se debe entender por "mejora" de la enseñanza de las matemáticas. 
Una manera implícita de responder a esta cuestión es considerar que la mejora consiste en seguir unas determinadas tendencias actuales sobre la enseñanza de la matemática, ya que se considera que la enseñanza realizada siguiendo estas tendencias es de calidad. Algunas de estas tendencias son específicas de la enseñanza de las matemáticas, mientras que otras son aplicables también a la enseñanza de otras materias.

Estas tendencias se pueden deducir de las publicaciones más relevantes del área por ejemplo, handbooks sobre la investigación en educación matemáticas (LESTER, 2007; ENGLISH, BARTOLINI-BUSI, JONES, LESH, y TIROSH, 2008), o publicaciones de la serie ICMI studies (STACEY, CHICK, KENDAL, 2004; BATANERO, BURRILL, READING, 2011) - de la creación de Topic Study Group, en los congresos (por ejemplo, el TSG4 New developments and trends in mathematics education at upper secondary level del ICME 11). Por otro lado, varios autores desde el campo de la enseñanza de las matemáticas han reflexionado sistemáticamente acerca de cuáles son las tendencias actuales en la educación matemática (MÜLLER, 2000; GUZMÁN, 2007; FONT, 2008). Estos autores, aunque presentan diferencias en las tendencias que consideran más relevantes, coinciden en una serie de tendencias comunes o al menos no contradictorias. Estas tendencias han tenido su impacto en las directrices oficiales en diferentes estados de Brasil - por ejemplo, las directrices del plan de estudios del estado de Paraná (2009) - y su influencia es evidente en los Parámetros Curriculares Nacionales (BRASIL, 2000). Una de las tendencias que coinciden mayoritariamente es la incorporación de nuevos contenidos.

\section{La incorporación de nuevos contenidos de las matemáticas en la Educación Básica y los procesos de instrucción}

Hay una tendencia a incorporar nuevos contenidos de geometría, estadística y probabilidad y matemáticas discreta. La necesidad de una recuperación de los contenidos geométricos en la enseñanza matemática es algo en lo que todos los interesados en la enseñanza de las matemáticas parecen coincidir. También se observa la tendencia al aumento de los contenidos de estadística y probabilidad. Esta es una tendencia en la que todos los sistemas educativos parecen concordar y efectivamente son muchos los países que incluyen en sus programas de enseñanza secundaria estas materias. También se considera que determinadas partes de la Matemática Discreta son lo suficientemente elementales como para poder formar parte con éxito de la enseñanza no universitaria. La combinatoria clásica, así como los aspectos modernos de ella, tales como la teoría de grafos o la geometría combinatoria, junto a la teoría elemental de números se consideran los candidatos más adecuados.

La mejora en la enseñanza de las matemáticas se concreta en un proceso de instrucción, lo que implica, entre otras cosas, la planificación, la implementación y rediseño de una secuencia de tareas para ser trabajada en una clase en particular. 
La primera fase de un proceso de instrucción es el diseño de una secuencia de tareas para enseñar un determinado tema. Esta secuencia debe tener en cuenta: un tiempo determinado, un público al que se destina y, preferiblemente, las actividades propuestas de manera detallada. La segunda fase es la implementación de la planificación. Por último, la tercera etapa es la evaluación del proceso de instrucción implementado $y$, si es el caso, su rediseño.

Según Godino, Contreras \& Font (2006), tanto la planificación como en implementación de un proceso de instrucción de un contenido matemático, implica una secuencia de configuraciones didácticas. Estas configuraciones incluyen tres aspectos: (i) una configuración epistémica, se refiriere a una tarea, los procedimientos para su resolución, el lenguaje, los conceptos, las proposiciones, los argumentos; (II) una configuración instruccional, es la red de aspectos docentes, discentes y mediacionales puestos en juego en la resolución de la tarea; (III) una configuración cognitiva, red de conocimientos personales (conocidos y/o aprendidos) que se ponen en juego en la implementación. Por otro lado, Godino, Font, Wilhelmi y Castro (2009), argumentan que una fase importante de los procesos de instrucción es su evaluación y rediseño mediante criterios de idoneidad (BREDA, FONT y LIMA, 2015), lo que permite centrarse en la mejora de futuras implementaciones.

\section{Metodología del estudio}

Se ha utilizado una metodología de investigación cualitativa, de acuerdo con Ludke y André (1986), que se basa en la comprensión y interpretación de los datos. En una primera fase se consideraron dos aspectos cualitativos, el primero es el tipo de innovación y el segundo es la fase del proceso de instrucción contemplado (planificación, implementación y rediseño).

Para ello, se seleccionaron veintinueve TCC realizados y publicados en el estado de Rio Gran del Sur, desde el primer semestre de 2013 hasta el segundo semestre de 2014. La selección se realizó de acuerdo con las siguientes etapas: en primer lugar, se hizo la distribución, por estados, de las universidades participantes del programa con el siguiente resultado: en trece estados participa una sola universidad, en cuatro estados participan dos universidades y en los otros estados participan tres o más universidades. En una segunda etapa, fue seleccionado el estado de Rio Gran del Sur, pues, por un lado, dicho estado participa del programa con de dos universidades (Universidad Federal de Santa María y la Universidad Federal de Río Grande), por otro lado presenta un numero de producciones finales razonable (veintinueve TCC) de manera que se puede inferir conclusiones y por último, otra razón de peso, fue la facilidad de acceso a los documentos seleccionados para el análisis. 


\section{Análisis de los resultados}

En relación a la cantidad de trabajos que incorporan la introducción de contenidos matemáticos de la enseñanza superior en la Educación Básica en sus propuestas de innovación didácticas, nos encontramos con que, de los 29 trabajos publicados, 13 proponen dicha incorporación (aproximadamente el cuarenta y cinco por ciento de los TCC del estado de Rio Gran del Sur).

En relación a los tipos de contenidos que se incluyen en estas propuestas se encuentran los siguientes: (i) contenidos relacionados con Matemática Discreta - presentes en cinco trabajos que abordan respectivamente el estudio de las Ecuaciones Diofánticas Lineales, Recurrencias Lineales, Inducción Matemática y Congruencias y Grupos -; (ii) introducción de contenidos relacionados con el Cálculo - presentes en tres TCC que abordan respectivamente el estudio de la idea de límites, cálculo del área bajo de curvas, concepto intuitivo de la derivada -; (iii) contenidos relacionados con la Geometría - presentes en dos trabajos que abordan, respectivamente, la geometría proyectiva y estudio de las curvas Mecánicas; (iv) un TCC que aborda el tema del teorema de Napoleón para la enseñanza de los números complejos; (v) un TCC que incorpora la ecuación de Van Der Waals para la enseñanza de las ecuaciones cúbicas y (vi), por último, otro que incorpora el uso de la idea del Método de Mínimos Cuadrados (MMC) para el estudio del producto matricial.

Aunque, de alguna manera, trece de los veintinueve trabajos finales de curso incorporen la introducción de nuevos contenidos matemáticos en sus propuestas, sólo nueve de ellos presentan alguna de las fases del proceso de instrucción (planificación, implementación y rediseño). La mayoría (siete de ellos) contemplan la planificación y la implementación, dos tienen solamente la planificación y ningún de ellos presenta rediseño de la secuencia didáctica. A continuación, en el cuadro 1 , por cuestiones de espacio, se presentan solo los resúmenes de los dos trabajos finales de curso que presentan la fase de planificación del proceso de instrucción. 


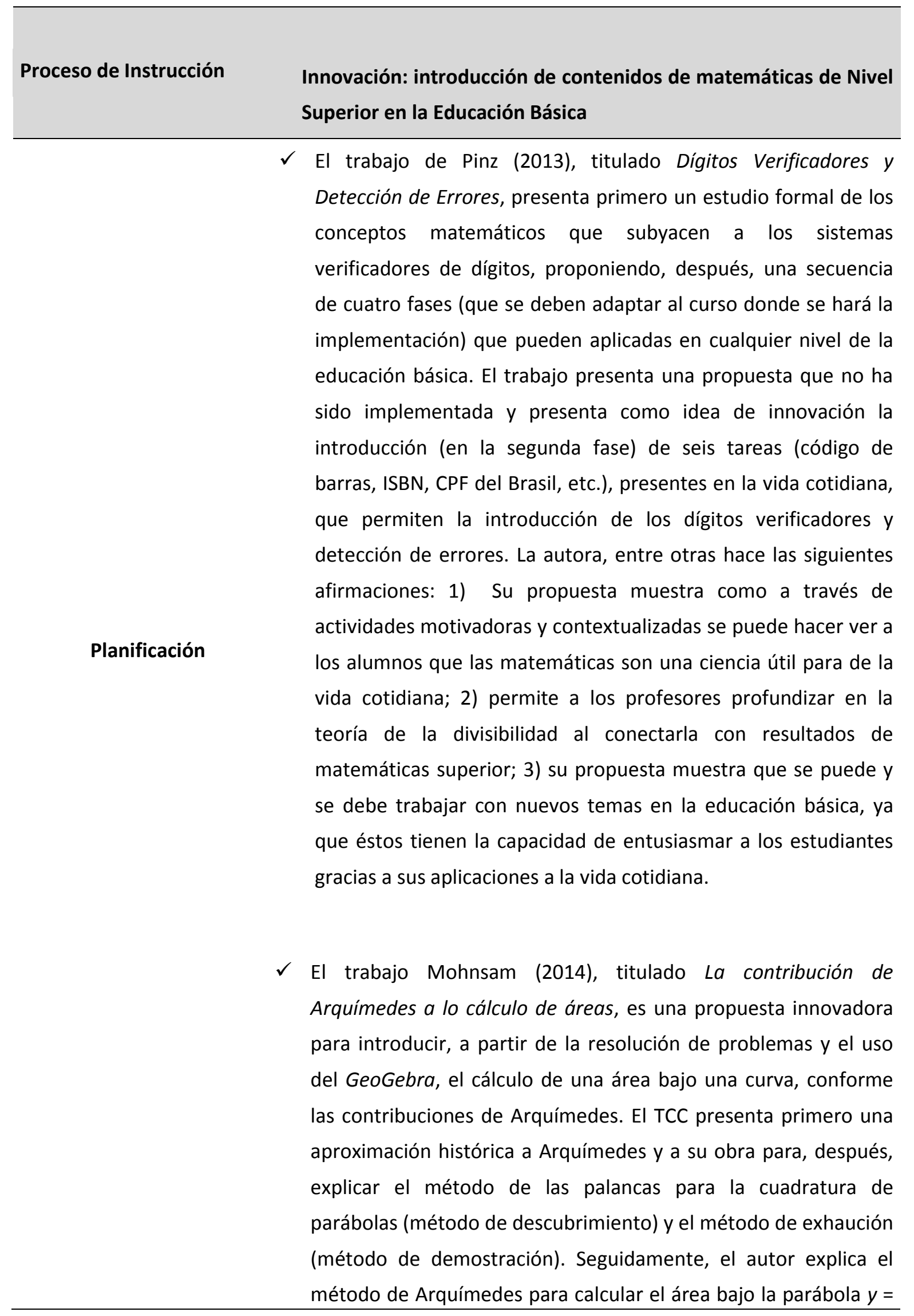

142 DOI: Em andamento.

R. Bras. de Ensino de C\&T 
$x^{2}$, entre 0 y un valor $b$ (entero), y lo compara con los métodos usados por Pascal, Fermat y Riemann. También dedica un capítulo a realizar estimaciones de error en los cálculos de áreas bajo de curvas que muestran, que si se aumenta el número de divisiones y se añaden más rectángulos el resultado es más preciso. El autor concluye que las ideas de Arquímedes son intuitivas $y$, en consecuencia, pueden ser utilizados por los estudiantes de secundaria para estudiar el área bajo una curva si se utilizan recursos computacionales como el Geogebra. En concreto, propone tareas que permiten ver a los alumnos que en el cálculo aproximado de áreas limitadas por curvas, que son gráficos de funciones, si se aumenta el número de rectángulos interiores y exteriores se obtiene una aproximación cada vez más precisa.

(Fuente: nuestra autoria.)

\section{Las justificaciones dadas a la incorporación de contenidos de matemáticas de la educación superior en la educación básica}

Resulta relevante conocer qué tipo de argumento dan los autores de los trabajos para justificar que la incorporación de contenidos de matemáticas de la educación superior en la educación básica que ellos hacen en sus TCC representa un mejora en la enseñanza en las matemáticas.

Dos de los trece trabajos afirman que incorporar contenidos de educación superior en la Educación Básica - sobre todo el contenido relacionado con Matemática Discreta y la recuperación de teoremas y procedimientos sugeridos por la historia de las matemáticas - mejora la enseñanza de las matemáticas, ya que aumenta el conocimiento de los estudiantes, ayuda a construir conceptos y entusiasma a los estudiantes.

Pero también se fortaleció la convicción de que podemos y debemos trabajar con "nuevos temas" que están, o bien no están, directamente relacionados con el currículum. Pues ellos tienen la capacidad de entusiasmar a los estudiantes, los despiertan, y amplían sus conocimientos. (PINZ, 2013, p.44).

"Como las ideas de Arquímedes son intuitivas, podemos inferir que pueden ser utilizadas por los estudiantes que no estudiaran el cálculo integral en la escuela media, para construir la noción de área de una manera más general." (Mohnsam, 2014, p. 73). 
Siete de los trece trabajos consideran que la incorporación de contenidos matemáticos de la enseñanza superior en la Educación Básica - relacionados con recurrencia, la inducción matemática, introducción al cálculo diferencial e integral, la geometría proyectiva, ecuaciones cúbicas y método de mínimos cuadrados - son una mejora de la enseñanza de las matemáticas porque, según los autores, en general se propicia: 1) la activación de procesos relevantes, como la modelación de situaciones extramatemáticas; 2) la construcción de conceptos y establecimiento de relaciones intramatemáticas, por ejemplo al relacionar los contenidos de nivel superior con contenidos de la Educación Básica; 3) la transición con los estudios universitarios posteriores al tener el alumno una idea intuitiva de contenidos que se estudian en la universidad.

"En este trabajo mostramos cómo el razonamiento recursivo está presente en nuestra vida cotidiana y cómo debe ser utilizado en el aula con el fin de hacer las clases más atractivas." (Martins, 2014, p. 61).

"[...] La parte la geometría proyectiva que permite un diálogo con la escuela primaria es, precisamente, el fundamento matemático de las reglas de diseño en perspectiva - campo que la Geometría Proyectiva comparte con la Geometría Descriptiva [...] En el contexto escolar, podrían reanudarse debates sobre las Geometrías no Euclidianas, comparando el postulado de las paralelas en la Geometría Euclidiana, con el postulado de la Hiperbólica y de la Elíptica; (...) podríamos explorar las proyecciones ortogonales de poliedros más complejos, cuerpos redondos y objetos cotidianos; sobre las transformaciones geométricas, podríamos explorar el carácter proyectivo de isometrías y homotecias." (Gonçalves, 2013, p. 21 e 147).

"El principal objetivo de este estudio fue verificar la posibilidad de introducir en el tercer año de la secundaria el Método de Mínimos Cuadrados, normalmente enseñado en la educación superior. La elección de este tema se debió a la poca o nula aplicación que se le da al producto de matrices en la escuela secundaria." (Souza, 2014, p. 68).

"Actividades diseñadas con el objetivo de introducir estos conceptos intuitivos (relacionado con el cálculo) en la escuela secundaria pueden aumentar la mirada del estudiante, durante el estudio de funciones, de forma que la introducción de estas ideas intuitivas, facilitan sus estudios posteriores." (Molon, 2013, p. 112).

"[...] Reforzamos la importancia de temas como Relaciones de Recurrencia y la Inducción Matemática en la Enseñanza de las Matemáticas, ya que el primero está conectado directamente a las computadoras y los temas ya estudiados como PA y PG. El segundo, pues, desarrolla la idea de infinito, que es un 
concepto un tanto difícil para la comprensión de los estudiantes." (Souza, 2013, p. 48).

"[...] La enseñanza de las matemáticas avanzada se puede introducir en la escuela secundaria, con muchos beneficios para el estudiante, tanto en la construcción geométrica, como en la aritmética." (Lopes, 2014, p. 104).

En general, las justificaciones dadas para este tipo de innovación en la Educación Básica son que la incorporación de contenidos de nivel superior permite: 1) nuevas formas de relacionar y acercar los contenidos matemáticos, 2) la realización de procesos relevantes (por ejemplo, la generalización, la modelación de situaciones extramatemáticas, establecimiento de relaciones intramatemáticas, de significación), 3) clases más atractivas que despiertan el interés de los estudiantes.

\section{Consideraciones finales}

Este estudio tiene como objetivo comprender el papel de la incorporación de contenidos de matemáticas de la educación superior en la Educación Básica en los trabajos de conclusión del curso realizados en el Máster Profesional en Matemáticas en la Red Nacional. Al analizar las veintinueve obras publicadas en Rio Gran del Sur, se concluye que el cuarenta y cinco por ciento de los TCC incorporan contenidos de las matemáticas de nivel superior en sus propuestas didácticas, especialmente contenidos de Matemáticas Discreta y de Cálculo.

Los nueve TCC que contemplan alguna modalidad de proceso de instrucción en sus propuestas (planificación, implementación o rediseño), permiten inferir que los profesores entienden la innovación de la enseñanza de las matemáticas en términos de un cambio de contenido matemático. Dicho cambio, según los profesores, permite enseñar una matemática de mayor calidad, donde la calidad, en la mayoría de los casos, se entiende en términos de mayor riqueza de procesos matemáticos, entendida "[...] en el sentido de que la resolución de las tareas propuestas conlleva la realización de procesos relevantes en la actividad matemática, como son la argumentación, la resolución de problemas, la modelización y, en particular, el proceso de conexión". (FONT, 2015, p. 45).

\section{Referencias}

BATANERO, C., BURRILL, G. \& READING, C. Teaching Statistics in School Mathematics-Challenges for Teaching and Teacher Education. ICMI/IASE Study: The 18th ICMI Study, 2011.

BRASIL. 2000. Parâmetros Curriculares Nacionais do Ensino Médio, 2000. Disponível em: http://portal.mec.gov.br/seb/arquivos/pdf/blegais.pdf. Acesso em nov. 2014.

R. B. E. C. T., vol 8, núm. 3, mai-ago.2015 ISSN - 1982-873X

DOI: Em andamento. 
BRASIL. 2013a. Uma análise quali-quantitativa de perfis de candidatos ao Mestrado Profissional em Matemática em Rede Nacional (PROFMAT). Relatório final do procedimento de análise qualiquantitativa de perfis de candidatos e aprovados no Mestrado Profissional em Matemática em Rede Nacional (PROFMAT), SBM, 2013a.

BRASIL. 2013b. Avaliação suplementar externa do programa de mestrado profissional em matemática em rede nacional (PROFMAT), CAPES, 2013.

BRASIL. 2014. Lei № 13.005. Aprova o Plano Nacional de Educação (PNE) e dá outras providências. Ministério da Fazenda (MF); Ministério do Planejamento, Orçamento e Gestão (MP); Ministério da Educação (MEC), 25 de junho de 2014.

BREDA, A., FONT, V. y LIMA, V. M. R. A noção de idoneidade didática e seu uso na formação de professores de matemática. Jornal Internacional de Estudos em Educação Matemática, v. 8, p. 441, 2015.

ENGLISH, L. D., BARTOLINI-BUSI, M., JONES, G. A., LESH, R. and TIROSH, D. Handbook of International research in mathematics education. London: Lawrence Erlbaum Ass, 2008.

FONT, V. Enseñanza de las Matemáticas. Tendencias y perspectivas. En Gaita, C. (Ed.), Actas del III Coloquio Internacional sobre Enseñanza de las Matemáticas, p. 21-62. Lima, Perú: Pontificia Universidad Católica del Perú, 2008.

GODINO, J.D.; CONTRERAS A.; FONT, V. Análisis de procesos de instrucción basado en el enfoque ontológico-semiótico de la cognición matemática. Recherches en Didactique des Mathématiques, 26 (1), 39-88, 2006.

GODINO, J. D., FONT, V., WILHELMI, M. R. \& CASTRO, C. Aproximación a la dimensión normativa en didáctica de las matemáticas desde un enfoque ontosemiótico. Enseñanza de las Ciencias, 27(1), 59-76, 2009.

GONÇALVES, T. S. Uma Introdução à Geometria Projetiva para o Ensino Fundamental. 2013. 149f. Dissertação (mestrado) - Mestrado Profissional em Matemática em Rede Nacional PROFMAT, Instituto de Matemática, Estatística e Física, Universidade Federal do Rio Grande, Rio Grande, 2013.

GUZMÁN, M. (2007). Enseñanza de las ciencias y la matemática. Revista Iberoamericana de Educación, 43, 19-58.

LESTER, F. Second handbook of research on mathematics teaching and learning. Greenwich, Connecticut: Information Age Publishing, Inc. y NCTM, 2007.

LOPES, A. C. Um relato sobre à introdução às somas de Riemann na Educação Básica. 2014. 109

f. Dissertação (mestrado) - Mestrado Profissional em Matemática em Rede Nacional - PROFMAT, Centro de Ciências Exatas, Universidade Federal de Santa Maria, Santa Maria, 2014.

146 DOI: Em andamento.

R. Bras. de Ensino de C\&T 
LUDKE, M. \& ANDRÉ, M. E. D. A. Pesquisa em educação: abordagens qualitativas. São Paulo, Editora Pedagógica e Universitária, 99p, 1986.

MARTINS, T. E. Equações de Recorrência na Educação Básica. 2014. 74f. Dissertação (mestrado) Mestrado Profissional em Matemática em Rede Nacional - PROFMAT,Instituto de Matemática, Estatística e Física, Universidade Federal do Rio Grande, Rio Grande, 2014.

MOHNSAM, J. C. As contribuições de Arquimedes para o cálculo de áreas. 2014. 86f. Dissertação (mestrado) - Mestrado Profissional em Matemática em Rede Nacional - PROFMAT,Instituto de Matemática, Estatística e Física, Universidade Federal do Rio Grande, Rio Grande, 2014.

MOLON, J. Cálculo no Ensino Médio: uma abordagem possível e necessária com o auxílio do software GeoGebra. 2013. 195f. Dissertação (mestrado) - Mestrado Profissional em Matemática em Rede Nacional - PROFMAT, Centro de Ciências Exatas, Universidade Federal de Santa Maria, Santa Maria, 2013.

MOREIRA, M. A. O mestrado (profissional) em ensino. RBPG. Revista Brasileira de PósGraduação, Brasília, v. 1, n. 1, p. 131-142, 2004.

MÜLLER, I. Tendências atuais de Educação Matemática. UNOPAR Cient., Ciênc. Hum. Educ., Londrina, v. 1, n. 1, p. 133-144, 2000.

PARANÁ. 2009. Diretrizes curriculares de matemática para a educação básica. SEED: Curitiba, 2009.

PINZ, C. R. F. Dígitos Verificadores e Detecção de Erros. 2013. 53f. Dissertação (mestrado) Mestrado Profissional em Matemática em Rede Nacional - PROFMAT, Instituto de Matemática, Estatística e Física ,Universidade Federal do Rio Grande, Rio Grande, 2013.

RONDERO, C. \& FONT, V. Articulación de la complejidad matemática de la media aritmética. Enseñanza de las Ciencias, №. 33.2, p.29-49, 2015.

SOUZA, M. E. D. Uma proposta de abordagem ao problema de Flávio Josefo aplicada ao Ensino Médio. 2013. 61f. Dissertação (mestrado) - Mestrado Profissional em Matemática em Rede Nacional - PROFMAT, Centro de Ciências Exatas, Universidade Federal de Santa Maria, Santa Maria, 2013.

SOUZA, S. A. Estudo do produto matricial por meio do Método dos Mínimos Quadrados: uma abordagem destinada ao Ensino Médio. 2014. 87f. Dissertação (mestrado) - Mestrado Profissional em Matemática em Rede Nacional - PROFMAT, Centro de Ciências Exatas, Universidade Federal de Santa Maria, Santa Maria, 2014.

STACEY, K., CHICK, H. \& KENDAL, M. The Future of the teaching and learning of algebra. Dordrecht: Kluwer A. P., 2004. 
Adriana Breda, Doutoranda em Educação em Ciências e Matemática pela Pontifícia Universidade Católica do Rio Grande do Sul (PUCRS). E-mail: adriana.breda@gmail.com.

Vicenç Font, Doutor em Filosofia e Ciências da Educação pela Universidade de Barcelona (UB). Professor Titular do Departamento de Didática das Ciências Experimentais e Matemática da Universidade de Barcelona (UB), Barcelona, Espanha. E-mail: vfont@ub.edu.

Valderez Marina do Rosário Lima, Doutora em Educação pela Pontifícia Universidade Católica do Rio Grande do Sul (PUCRS). Professora do Programa de Pós-Graduação em Educação em Ciências e Matemática da Pontifícia Universidade Católica do Rio Grande do Sul (PUCRS). E-mail: valderez.lima@pucrs.br. 